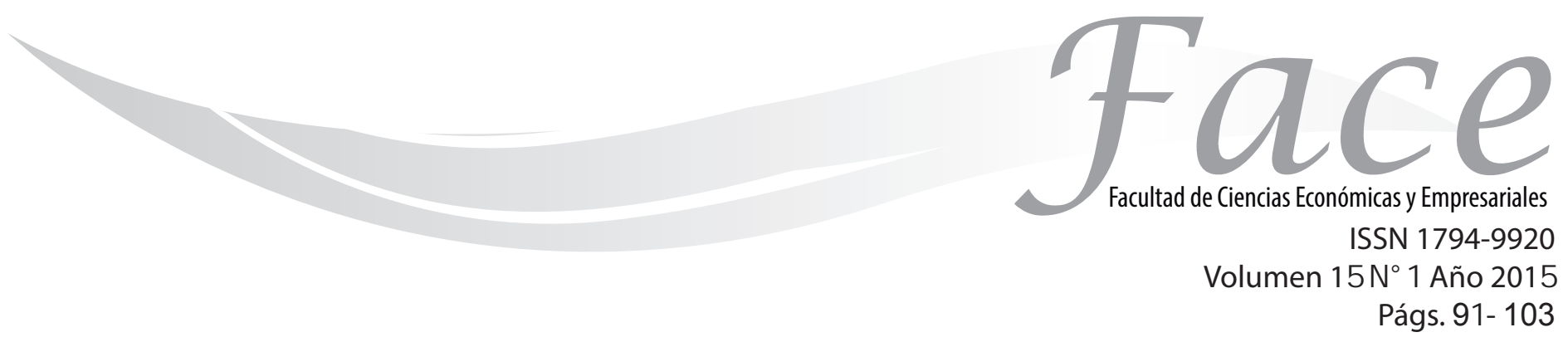

\title{
EXPERIENCIAS DEL BRANDING COMO ESTRATEGIA PARA EL POSICIONAMIENTO EN EL MERCADO INTERNACIONAL.
}

Fecha de Recepción: 26 de Marzo 2015

\author{
Liliana Marcela Bastos Osorio* \\ Johanna Milena Mogrovejo Andrade** \\ Lucy Gómez Mina***
}

Fecha de Aprobación: 25 de Junio 2015

\begin{abstract}
Resumen:
Este trabajo tuvo por objetivo central realizar una reflexión sobre las estrategias del Branding que han sido aplicadas desde los diferentes énfasis: económico, deportivo y educativo, con el fin de posicionar una marca a nivel nacional e internacional. Se hizo una revisión documental y bibliográfica del concepto de autores como Rob Frankel e Interbrand y de los diferentes estudios realizados aplicando las estrategias del Branding, Palacio y Urbina (2014), Brill Ramírez, y Pradenas, (2007) y Lema y Peláez (2009). Las principales conclusiones encontradas se tiene que con la implementación del Branding algunas empresas y sectores tienden a la internacionalización con la utilización de una marca. Es así que al observar el caso de la ciudad de San José de Cúcuta, con el desarrollo de la marca representativa del sector marroquinero se trató de destacar la importancia que tiene una marca apropiada, la cual permitirá la difusión de conceptos claros a través de los atributos y valores que posee el sector marroquinero de la ciudad.

Palabras claves: Branding, Marca, Mercado Internacional.
\end{abstract}

\footnotetext{
* Magíster en Administración (Universidad Nacional Experimental del Táchira, Venezuela), Especialista en Finanzas (Universidad Autónoma de Bucaramanga, Colombia) Profesor adscrito al Departamento de Estudios Internacionales y de Fronteras (Facultad de Ciencias Empresariales), Universidad Francisco de Paula Santander (Cúcuta, Colombia). Miembro del Grupo de Investigación (GIDSE) para el Desarrollo Socioeconómico. contacto: lilianamarcelabo@ufps.edu.co

** -Candidata a Doctor en Estudios Políticas (Universidad Externado de Colombia), Magíster en Administración (Universidad Nacional Experimental del Táchira, Venezuela), Especialista en Control Interno e indicadores de gestión (Universidad de Pamplona, Colombia) Profesor adscrito al Departamento de Estudios Internacionales y de Fronteras (Facultad de Ciencias Empresariales), Universidad Francisco de Paula Santander (Cúcuta, Colombia). Director del Departamento de Estudios Internacionales y de Fronteras y Director del programa de Comercio Internacional Universidad Francisco de Paula Santander (Cúcuta, Colombia), Director del Grupo de Investigación (GIDSE) para el Desarrollo Socioeconómico. contacto: johannamogrovejo@ufps.edu.co

*** Doctorado en educación (UNIVERSIDAD PEDAGOGICA EXPERIMENTAL LIBERTADOR GERVASIO RUBIO), Magister En Educación (Pontificia Universidad Javeriana - Puj - Sede Bogotá) Licenciatura En Ciencias de La Educación Español I, Docente Universidad Francisco de Paula Santander.Contacto: Lugomi60@hotmail.com
} 


\title{
EXPERIENCIAS DEL BRANDING COMO ESTRATEGIA PARA EL POSICIONAMIENTO EN EL MERCADO INTERNACIONAL
}

\begin{abstract}
:
The main objective of this work was to undertake a reflection on branding strategies that have been used with different emphasis in economics, sports and education, in order to position a brand at a national and international level. A documentary and bibliographic review of the concepts of Rob Frankel and Interbrand and various studies of authors such as Urbina \& Palace (2014), Brill, Ramirez \&Pradenas, (2007) and Lema\&Pelaez (2009) related to branding strategies was conducted. Findings showed that with the implementation of branding and the use of a trademark some companies and sectors tend to internationalize. It is so that, in the case of the city of San José de Cúcuta, with the development of a representative brand in the leather goods' sector the aim was to highlight the importance of an appropriate label, which will allow the dissemination of the attributes and values of the city's leather goods.
\end{abstract}

Keywords: Branding, brand, internationalmarket

\section{BRANDING EXPERIÊNCIAS COMO ESTRATÉGIA PARA O CARGO NO MERCADO INTERNACIONAL}

\begin{abstract}
Resumo:
O objetivo central do trabalho foi realizar uma reflexão sobre as estratégias do branding que foram utilizadasem diferentes áreas como economia, esportes e educação, a fim de posicionar uma marca a nível nacional e internacional.Realizou-se uma revisão bibliográfica e documental de autores como Rob Frankel e Interbrand, assim como, dediferentes estudos realizados sobre as estratégias do branding;Palacio\&Urbina (2014), Brill, Ramírez\&Pradenas, (2007) e Lema \&Peláez (2009). Os resultados mostram que com a aplicação do branding, algumas empresas e setores tendem a internacionalizar-se com a utilização de marca. É assim que,no caso da cidade de San José de Cúcuta, com o desenvolvimento de uma marca representativa do setor de couro tratou-se de destacar a importância que tem uma marca adequada, que permitirá a divulgação dos atributos e valores que tem o couro da cidade.
\end{abstract}

Palavras-chave: Branding, Marca, Mercado Internacional 


\section{Introducción:}

Actualmente, las empresas están en la búsqueda de consolidar las preferencias de los consumidores hacia los productos fabricados por ellos, de tal manera que estos bienes y servicios se posesionen no solo en la mente sino en el corazón de los consumidores. De allí es que nace el concepto de marca, y por tanto los empresarios están interesados en crear valor a la marca. El Branding es una herramienta para poder alcanzar y establecer una marca no solo para bienes y servicios, sino que también para una ciudad, o para una persona. Con las estrategias de Branding se intenta establecer un proceso de construcción de una marca, la gestión de los activos vinculados a esa marca, ya sea de manera directa o indirecta. Una forma más clara de entender el Branding es el posicionamiento en el mercado que puede tener una marca y lo que la pueda diferenciar de otras.

El presente trabajo es una reflexión de las estrategias del Branding que han sido aplicadas a diferentes sectores, buscando posicionar el sector a nivel nacional e internacional y primordialmente en la parte emocional de los consumidores que al adquirir algún producto en cualquier parte del mundo, se sientan identificados con el mismo. Hoy en día es más difícil diferenciarse en el mercado, ya que las empresas lideran procesos de calidad y de mejoramiento de sus productos y están buscando en la minimización de los costos de producción. De esta manera, la clave de los negocios está en el Branding, el cual considera el poder de la marca, como un elemento diferenciador en la estrategia en el mercado.

Un valor agregado que tienen las empresas son las marcas fuertes, el cual en algunos casos tiene un mayor desempeño o es más dinámico que el mismo producto que la empresa produce. Las marcas tienen una característica muy especial y peculiar, la de impactar e influenciar en nuestra vidas y en la forma de ver mejor el mundo. Marcas grandes como Juan Valdez, Colombia CO, Totto entre otras, son muy reconocidas y tienen una alta representación de Colombia en el exterior. Es así como las marcas ya necesariamente no son vistas solo como valores agregados de las empresas sino que han pasado hacer parte de los activos de la misma.

\section{Metodología:}

La metodología aplicada en este artículo de reflexión fue la revisión documental y bibliográfica del concepto de marca, branding, de la evolución del mismo y de la aplicabilidad en los diferentes sectores económicos; autores como Rob Frankel y la Consultoría Internacional del Branding, Interbrand. De igual manera, se hizo una revisión de los diferentes estudios y trabajos de grados realizados aplicando las estrategias del Branding, en empresas nacionales e internacionales, como son el proyecto de investigación de Palacio y Urbina (2014), Brill Ramírez, y Pradenas, (2007), Lema y Peláez (2009), Hoyos, (2011), Aguirre, (2008), Cruz (2011), Ayala, (2009) y Carreño, (2013). Estos trabajos de investigación, pertenecen a diferentes sectores en la economía, y son estudios del ámbito internacional y de Colombia. Después de esta clasificación y selección, se realiza una reflexión teniendo en cuenta aspectos positivos y negativos de la aplicabilidad del branding en las diferentes tipos de empresas.

\section{Marco Teórico:}

\section{Branding}

La palabra Branding viene del vocablo inglés "brand" que significa marca. Por medio del significado de esta raíz etimológica, se puede decir que los productos o servicios que se han de ofrecer al mercado se pueden diferenciar de los demás, "marcándolos" con un sello específico que logre que estos sean reconocidos por la sociedad y creando una preferencia por encima de los demás productos y servicios en el mercado (Guzmán, 2010).

La teoría del Branding según Rob Frankel, desarrollador de acciones de branding, consiste en lograr que una marca sea percibida como la mejor, como la única solución, lo que se traduce en fidelidad del cliente hacia una marca y/o producto. Dicho en otras palabras, es el posicionamiento que se adquiere en el mercado, el cual se obtiene luego de un largo proceso en el que la compañía logra transmitir su personalidad a la mente del consumidor (Arango, 2009). 
Interbrand (2000), menciona que "el branding consiste en desarrollar y mantener el conjunto de atributos y valores de una marca de manera tal que sean coherentes, apropiados, distintivos, susceptibles de ser protegidos legalmente y atractivos para los consumidores.

Jez Frampton miembro de la Consultoría Internacional del Branding, conocida como Interbrand (2012) menciona formalmente la aplicación del Branding o todo el proceso desde la concepción hasta la consolidación de una marca se extendió a partir del siglo XIX, pero las marcas basadas en la reputación de los artesanos han existido a través de los siglos.

A través de la historia se ha observado la evolución que han tenido las marcas y la importancia que han ido adquiriendo para las empresas y para lo que representan en un mercado cada día más competitivo. Esto ha incidido a que diferentes autores se especialicen en el estudio de este fenómeno de manera amplia y a generar nuevos conceptos y nueva literatura sobre este tema. Por, lo tanto es importante revisar los primeros indicios que el tiempo nos presenta para conocer como inicia el punto de partida de toda esta revolución de las marcas.

En el curso de los siglos, las marcas y los logotipos fueron utilizados en un nivel local. Las excepciones eran las marcas distintivas utilizadas por reyes, emperadores y gobiernos. La flor de lis francesa, el águila de los Habsburgo en AustriaHungría y el crisantemo imperial en Japón indicaban propiedad o control. En la segunda mitad del siglo XIX, las mejoras en las comunicaciones y en los procesos de fabricación permitieron, por primera vez, la masificación de los productos de consumo, y muchas de las marcas más conocidas en la actualidad se remontan a ese período: las máquinas de coser Singer, los refrescos Coca-Cola, Bass Beer, los copos de avena Quaker, los viajes Cook, el jabón Sunligth, las películas kodak, los cheques de viaje American Express y los seguros Prudencial son ejemplos de ello. (Guzmán, 2010). .

Según Philip Kloter y Gary Armstrong (2008), autores del libro Fundamentos del Marketing, la Marca es el nombre, señal o diseño con el que se identifica y comercializa a un producto, bien o servicio de una empresa u organización y los diferencia del resto de la competencia. Es una combinación de elementos visuales y lingüísticos como logotipos, isotipos, imágenes, tipografías, gama cromática, iconos, nombres y slogans. Es el signo que utiliza un fabricante para indicar la proveniencia y autoría de los productos o servicios que ofrece. Por lo tanto la marca es un identificativo de origen. (Kloter, 2009).

"Las marcas, son los estándares que hacen reconocible a cada competidor, en esa lucha por las preferencias, de la clientela. Asimismo las marcas resultan ser iconos y representaciones visuales detrás de las cuales se manifiesta la promesa de una satisfacción hacia el cliente" (Nito, 2014).

Por otra parte Sánchez (2013) marca es todo signo susceptible de representación gráfica que sirva para distinguir en el mercado los productos o servicios de unas empresas de los de otra. Por lo tanto la define restrictivamente exigiendo que sean susceptibles de representación gráfica. (Sánchez, 2013).

Mientras en el aspecto publicitario de la marca se tiene un carácter agresivo, que es lo que permite al propietario conquistar el mercado por la sola autoridad del nombre dado al producto y no por el producto en sí mismo (Luna, 2011).

En entrevista con David Clifton, Director General de Interbrand México (Arango, 2009) da a conocer los cinco secretos del éxito de las marcas los cuales son:

- Relevancia de una marca: Debe brindar a los consumidores características notables y además, intentar en toda ocasión hablar en su mismo idioma. Es importante conocer a los clientes, quienes son, cómo son, qué quieren y cuál es la mejor manera de enviarles mensajes.

- Diferenciación de una marca: Debe encontrar atributos que la diferencien de las demás y también debe otorgar algo distinto a lo que hacen sus competidores.

- Credibilidad de marca: Implica que lo que promete una marca, siempre debe estar sustentado en la realidad del producto o del servicio. La credibilidad es un aspecto de suma importancia pues en ocasiones, las empresas de servicios lanzan estrategias de marca que no tienen nada que ver con las experiencias 


\section{LilianaMarcelaBastosCsorio-JdhennaMlenaMbgrovejoAndrade- LugyCónezMina}

del consumidor y la comunicación de la marca.

- Alcance: Las marcas con mayor éxito, han sido capaces de crecer más lejos de su ámbito natural, son capaces de conquistar segmentos casi imposibles para otros, rebasar fronteras geográficas, y extenderse mediante nuevas líneas de servicios o productos.

- Consistencia a lo largo del tiempo: Es importante lograr un posicionamiento de marca distintivo, realizando un análisis profundo de las necesidades de los consumidores y consistente con la cultura interna de la misma empresa (Arango, 2009).

Con estos factores de la marca se está proyectando directamente al consumidor una imagen propia que tiene una identidad y la mantiene vigente a lo largo del tiempo. Por ejemplo en cuanto a este tema de la imagen que proyecta una marca (Assael, 1987), menciona que esta representa percepciones generales formadas por información en una experiencia pasada. La imagen es contar con actitudes, creencias y preferencias, al obtener una imagen positiva es más agradable comprar la marca.

La imagen puede ser influenciada por varios factores incluyendo, la información, la promoción en periódicos, revistas, internet, de boca en boca entre otros, donde se juega un rol importante ya que gracias a esto se forma la imagen de un lugar o cosa (Luna, 2011).

El ciclo de las marcas según Kotler y Armstrong, en su libro fundamentos del marketing (2003) señalan que el ciclo de vida del producto (CVP) es el curso de las ventas y utilidades de un producto durante su existencia. Al tocar el tema de las marcas estas van más allá de sus identidades, las cuales responden a un contenido estratégico que les dan sentido, cualquier cambio de la identidad o de su contenido estratégico, produce directamente un cambio en la percepción del consumidor (La Base, 2009).
Figura 1.

Ciclo de vida de las Marcas.

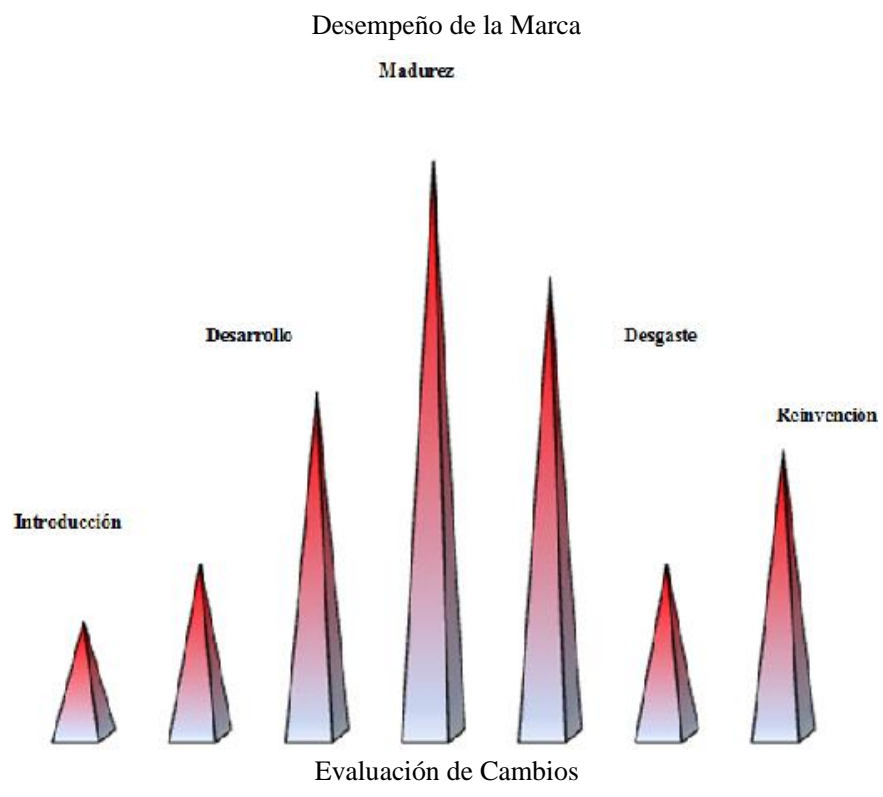

Fuente: Urbina y Palacios (2014)

El gráfico describe como, al igual que los productos, las marcas tienen un ciclo de vida: se crean, introducen, desarrollan, alcanzando así un nivel de madurez, que llegado un momento sufre un desgaste, el cual inevitablemente las conduce a un punto de decisión, que es la "Oportunidad o Muerte", donde los directivos de las empresas deben tomar la decisión de transformarla, reinventarla o bien dejarla morir. (Urbina y Palacios, 2014).

Se identifican las etapas por las cuales atraviesan las marcas durante su vida útil. Esto permite detectar el punto en el cual la marca se encuentra y así conducirla, re-direccionarla y mantenerla al punto de mayor valor (La Base, 2009).

\section{Experiencias Del Branding}

A partir de los años 80 la revolución de las marcas empieza a generar una tendencia para las empresas y su relación con el mundo del comercio y los negocios internacionales; $y$ en el cual se requiere de la construcción de una identidad propia de los productos elaborados por las empresas, representados por lo que se conoce hoy en día como el alma de toda empresa que es su marca. Es 
así que dentro del tema de marketing, se destaca el Branding, modelo que viene de la mano con toda esta revolución de los mercados y además con el cual se ha querido mostrar el modo de destacar un producto entre todos aquellos que recibe el cliente.

Dentro de este artículo de reflexión del Branding se hizo una exploración a algunos trabajos de investigación que buscan fortalecer e implementar dentro de su estrategia de nacionalización e internacionalización de sus productos este tema del Branding y de la marca.

Urbina y Palacios (2014) en su trabajo de grado "Estrategias branding para el sector marroquinero de San José de Cúcuta, Norte de Santander", plantean la creación de un concepto de imagen y marca propia que represente el sector de marroquinería de Norte de Santander en el ámbito nacional e internacional, ya que establecieron la necesidad que tiene el sector marroquinero para mostrarse, darse a conocer y proyectar una imagen sólida que lo posicione a nivel internacional y que refleje la calidad, la seriedad y las ganas con las cuales son elaborados los productos a comercializar.

El sector marroquinero no ha podido posicionar sus productos a nivel internacional debido a la falta de programas de investigación y desarrollo de nuevas tecnologías y productos, además, las dificultades existentes en la asociatividad, individualismo, deslealtad y falta de solidaridad entre los empresarios del sector. (J. Rangel, entrevista personal, noviembre de 2013 realizada por Urbina y Palacios).

De esta misma manera, dentro del estudio realizado por Urbina y Palacios (2014) determinan un diagnóstico de la importancia de la estrategia del Branding en las siguientes ideas:

- Los productos de la región no tienen una identidad de marca región establecida, hasta ahora se está iniciando con el proceso de internacionalización de las empresas.

- Las empresas en Cúcuta ya están llevando sus productos a mercados externos, pero estos productos no identifican nuestra región como tal, no existe esa marca región y es preciso lo que necesitamos.
- Problemas en este proyecto es lo que vamos a encontrar pero tenemos que solventarlos, y uno de ellos es el cómo llegar a proponer y sustentar esta marca y lo más importante que el empresario la acoja.

- Las universidades siguen cometiendo el error de esperar que el empresario sea quien lo busque, cuando son las universidades las que tienen que buscar al empresario y decirle, cual es mi oferta y en que les puedo ayudar a sacar sus productos tanto en el mercado nacional como internacional que sirva de fortaleza y que sea un valor agregado para el empresario. (Urbina y Palacios, 2014: 21)

Mediante el branding y la generación de una marca representativa del sector marroquinero los clientes han de reconocer y establecer con ella una relación emocional determinada: a través de esta marca se podrá comunicar seguridad, familiaridad, singularidad, diferencia, generando no sólo una memoria individual, sino también colectiva.

En definitiva en las últimas décadas se ha entendido que las marcas deben establecer una conexión emocional con el cliente y por lo tanto se quiere que los empresarios y los productos que estos llevan a los mercados internacionales vayan respaldados por una imagen que refleje dicha conexión. A través de la aplicación de las estrategias branding en el sector marroquinero, se pretende que sus empresarios puedan llegar a los sentimientos y a los deseos más profundos de los clientes a los cuales están dirigidos sus productos.

Una de las intenciones finales del desarrollo del proyecto de Urbina y Palacio (2014) fue la falta de cooperación entre los empresarios que lo conforman, factor que se ha pensado es fundamental para el desarrollo de estrategias que generen mayor competitividad al penetrar mercados internacionales, por lo tanto se ha creído que a través del establecimiento de una imagen que lo represente también se contribuirá con el acercamiento y complementación de los empresarios que pertenecen a este sector.

Otro trabajo de investigación es el de Ayala, J., el cual realizó un trabajo de grado con título: "Esquema de posicionamiento y persuasión para la marca LDU Liga Deportiva Universitaria a nivel nacional, tomando como plan piloto la ciudad de 


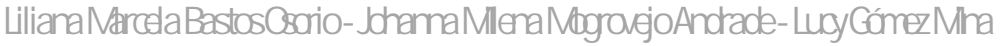

Ibarra" - Quito, Se fundamenta teóricamente en temas acerca del marketing, el branding, la marca como producto, su utilidad y desarrollo, se pretende colocar a LDU como una marca fuerte en el mercado, que la gente respete, la reconozca y perciba los privilegios de identificación y diferenciación de la misma (Ayala Rivero, 2009).

Esta investigación propone un esquema de posicionamiento y persuasión para la marca LDU a nivel nacional. Se tuvo en cuenta la ciudad de lbarra por cuanto no posee un equipo local y la hinchada se encuentra dividida. Se pretende colocar a LDU como una marca fuerte en el mercado, que la gente respete, la reconozca y perciba los privilegios de identificación y diferenciación de la misma.

La marca LDU Liga Deportiva Universitaria a nivel nacional se fundamenta teóricamente en temas acerca del marketing deportivo, el branding, la marca como producto, su utilidad y desarrollo. Se caracteriza sobre el equipo LDU, sus antecedentes, historia y acciones de marca realizadas a través del tiempo. Estos elementos claves marcan las directrices de la investigación que emplea técnicas como encuestas y entrevistas dirigidas a directivos de marketing y finanzas de LDU, hinchas, periodistas deportivos y públicos.

Del procesamiento de los datos obtenidos se logra estructurar un esquema novedoso, en el cual se combina el marketing mix y una estrategia comunicacional que garantiza un posicionamiento y persuasión para la marca LDU a mediano y largo plazo. Por lo cual este esquema es factible de aplicarlo, es original por cuanto no existen antecedentes que otros equipos hayan aplicado en forma técnica este tipo de estrategias y finalmente la marca obtiene réditos económicos.

Liga de Quito necesita expandir su hinchada a nivel nacional para así obtener la mayor cantidad de adeptos y socios, y posicionarse como el club con mayor hinchada en el país a largo plazo. Considerando que la mayoría de hinchas se localizan en Quito, no se ha podido ampliar de una manera efectiva y correcta el número de adeptos a nivel nacional y sobrepasar al que actualmente lidera la más grande hinchada (Barcelona Sporting Club ó Real Madrid). Si esta situación no se atiende oportunamente será muy difícil para el equipo de LDU alcanzar este objetivo.
Recorrer progresivamente las ciudades del país, para captar más adeptos con un esquema de posicionamiento, es el primer paso para lograr el objetivo con el cual LDU llegue a ser el número uno. En este caso se utilizará como punto de partida la ciudad de lbarra y se estima que a largo plazo, se puede llegar a todas las ciudades y provincias del país.

Peter Brill y Mauricio Pradenas realizaron un proyecto de título: "Branding en Chile, Universidad de las Américas. En esta investigación se desarrolló y estudio la influencia que ejerce el Branding al crear valor de una marca, cómo ellas son un recurso fundamental en las empresas ubicadas en este país, y mostrar la importancia que cumplen para el desarrollo adecuado de los procesos estratégicos de toda organización (Brill Ramírez, y Pradenas, 2007).

En el desarrollo del trabajo de investigación, se describe el caso de CACHANTUN, JUMBO, NESCAFÉ $Y$ MUNICIPALIDAD DE PROVIDENCIA en Chile. La finalidad de los autores, Brill y Pradena (2007), es lograr con estos casos que se presentan, una realidad distinta a la que se conoce tradicionalmente, dando a conocer la real e importante función del Branding en el proceso de posicionamiento de una Marca, mostrando como esta disciplina logra que una empresa sea exitosa en el mercado a través de su Marca, fortalecerla y crearle una imagen positiva para que de esta forma se pueda internacionalizar.

Estas cuatro empresas, tienen una Marca e Imagen Corporativa fuerte, la cual fue lograda a través del Branding durante los últimos años. Este caso se ha dado en el mercado nacional y ha servido como ejemplo para aquellas empresas que invierten grandes sumas de dinero para posicionarse dentro de un mercado y finalmente obtienen buenos resultados, lo que demuestra que no hay que dejar de lado conceptos como cultura, identidad e imagen corporativa, puede afectar de manera importante en el momento de querer posicionarse a través de su imagen de marca. (Brill y Pradena, 2007)

En el caso de la Municipalidad de Providencia, se enfrenta al paradigma de que "lo nuevo es mejor". En relación al territorio habitado, esto se traduce en una migración desde comunas establecidas a comunas nuevas, buscando calidad de vida y a veces status, con urbanizaciones "de moda". Este 


\section{Volunen15N1,Año2015}

fenómeno, característico en América del Sur, no se observa en ciudades europeas donde sus habitantes viven orgullosos, por generaciones, en la misma casa, en la misma calle. Para los grupos de elite y las nuevas clases emergentes, pareciera que las comunas se desgastan, se deterioran, envejecen, disminuyen su atractivo y finalmente, pierden valor.

Sin embargo, hay formas de rescatar el valor de vivir en una comuna consolidada. En Manhattan, barrios como Greenwich Village se re-valoraron y ganaron un nuevo prestigio atrayendo a grupos influyentes. Para Providencia, esto implica la necesidad de destacar sus valores a través de un adecuado trabajo de Branding para mantenerse vigente y atractiva. En lo más inmediato, se enfrenta un período de preparación para elecciones municipales, que necesariamente será competitivo en lo comunicación, dificultando los esfuerzos de Branding de la comuna. (Brill y Pradena, 2007)

Otro proyecto de aplicación del Brandig es el Francy Milena Aguirre (2008). Realiza el "Diseño de un plan de marketing estratégico para empresas de servicios temporales EST basado en el diagnóstico de medianas y grandes empresas del sector comercial" - Universidad Nacional de Colombia sede Manizales. La investigación permitió vislumbrar el panorama del sector de Empresas de Servicios Temporales "EST" en Pereira y Dosquebradas, orientados en un diagnóstico general del sector y logrando una acercamiento con las empresas comerciales medianas y grandes de las ciudades, esto con el fin de determinar los motivos reales por los cuales utilizan este tipo de servicios, los criterios más importantes a la hora de comprar y quien toma la decisión final de adquirir el servicio (Aguirre, 2008).

La investigación de Aguirre (2008), permitió vislumbrar el panorama del sector de Empresas de Servicios Temporales "EST" en Pereira y Dosquebradas, orientados en un diagnóstico general del sector y logrando una acercamiento con las empresas comerciales medianas y grandes de las ciudades en mención, esto con el fin de determinar los motivos reales por los cuales utilizan este tipo de servicios, los criterios más importantes a la hora de comprar, quien toma la decisión final de adquirir el servicio, que tan influyentes son los grupos de referencia, entre otros; esto se logra a través del análisis de diversas variables, las cuales fueron tomadas del comportamiento del consumidor organizacional y otras propias del sector en estudio.

Dichas variables dan fundamento al Plan de Marketing Estratégico propuesto en la presente investigación, el cual da respuesta a los intereses manifestados por las empresas comerciales medianas y grandes de Pereira y Dosquebradas. Dicho Plan de Marketing interrelaciona las estrategias, tácticas y actividades que se deben aplicar por parte de las Empresas de Servicios Temporales para lograr la efectiva comercialización de este tipo de servicio, igualmente hace énfasis en la forma como debe mejorar el posicionamiento de la actividad como tal, la cual se ha visto afectada por diferentes razones.

Natalia Lema y Adriana Peláez, realizaron un trabajo de grado titulado: Marcas en cuerpo y alma "las Marcas cambian de piel, pero no pierden su esencia"- Pontificia Universidad Javeriana, Bogotá Colombia. Resaltan como las compañías buscan establecer lazos emocionales con sus consumidores, estos al adquirir un producto es un proceso racional pero al momento de elegir una marca se convierte en un proceso emocional (Lema y Peláez, 2009). A través de este proyecto se intenta plantear el manual de identidad corporativa para la empresa de maquinaria de diagnóstico automotriz MACO S.A.

La investigación presentó una inmensa similitud en el comportamiento de las diferentes categorías del mercado. Se pudo observar que existe un temor frente al cambio y a la diferenciación. Todos los logos son diferentes pero manejan una gama cromática casi idéntica. Piense en la categoría de bancos y verá que todas tiene el color azul. Es hora de cambiar un poco y arriesgarse. Según Claudio Arango, gerente de Brandingdang, "la idea es más que seguir la corriente, romperla". (Lema y Peláez, 2009)

Las opiniones de los expertos arrojan un grado de similitud principalmente en la idea de plantear una estrategia para la creación de una marca o para su renovación. Sus creencias son coherentes y concuerdan a pesar de existir diferentes puntos de vista. La teoría y la práctica concuerdan en muchas opiniones y no son muchas las diferencias que se imponen. Sin embargo, mientras David Aacker aporta el proceso completo, de cómo construir una marca de manera sistemática, paso a paso, desde el 


\section{LilianaMbrcelaBastosGorio-J dhennaMlenaMbogovejoAndade- Lucy CónezMna}

nombre hasta la estrategia. Claudio Arango, Álvaro Arango, José Luis Arroyave, hablan un poco más de la evolución de la marca y no tanto del proceso sino de la importancia de la marca en las vidas de las personas y como se crean estilos de vida a partir de éstas. (Lema y Peláez, 2009)

Finalmente, teniendo en cuenta todos los aspectos que conforman una identidad visual, realizaron el manual de identidad corporativo para la empresa MACO S.A. Los autores consideran la oportunidad de hacer un cambio de imagen visual porque era una marca que llevaba treinta años en el mercado y no había sido renovada desde entonces, lo cual invitó a realizar un cambio innovador para darle un aire más moderno a la marca pero manteniendo su esencia. Para la realización del proyecto trabajaron de la mano con el gerente de la empresa y los empleados de la misma para entender como estaba siendo percibida la marca a nivel interno y externo y de esta manera, atender las necesidades que se presentaban para proyectarlas en una nueva imagen visual.

Diana Hoyos Forero, realizó una pasantía de título "Construcción de la identidad de marca para la empresa calzado ROGGER'S LTDA. - Universidad Autónoma del Occidente - Valle del Cauca Colombia. Al crear la identidad de marca de Calzado Rogger's, esta se refleja en sus comunicaciones para obtener una imagen de marca única percibida por sus públicos objetivos, direccionada de acuerdo a la imagen deseada por la empresa y a los objetivos corporativos (Hoyos, 2011).

Para el desarrollo de este trabajo se realizó una investigación previa de la empresa; se hizo una evaluación del estado actual de la marca en el mercado, conociendo sus percepciones, posicionamiento y antecedentes de comunicación de marca; el argumento en el que se desarrolla el proyecto, teniendo en cuenta la competencia, el mercado; el target al que se dirige, realizando un trabajo de campo con el público interno y externo de la empresa, finalizando con la entrega de la identidad de marca adecuada para la empresa y los públicos a los que se dirige esta.

La empresa calzado ROGGER'S LTDA cuenta con un diseño gráfico de marca que ha sido modificado en 3 ocasiones, manejando actualmente en sus comunicaciones dos de ellos, perdiendo por completo una linealidad de marca $y$ un direccionamiento de la imagen deseada. Durante su trayectoria Calzado Rogger's no ha tenido en cuenta el desarrollo de identidad de marca de acuerdo a las necesidades que la empresa o su público tienen. Debido a esto, no hay una herramienta que ayude a medir el impacto de la comunicación, ni el desarrollo de estrategias a futuro, ya que no existen bases para ello. (Hoyos, 2011)

Jonnathan Jair Cruz, realizó un trabajo de grado con título: "Estado actual del branding en las compañías spin off y start up académico: caso universidades públicas en Colombia - Universidad Nacional de Colombia, Bogotá. Estableció el proceso cíclico de branding para las empresas provenientes de la investigación académica, el cual comprende tres fases: la definición de la estrategia de marca, el diseño de marca (identidad) y la construcción y desarrollo de marca (imagen). El proceso de branding debe ser cíclico, ya que la retroalimentación de los resultados de la etapa de construcción y desarrollo de marca es un factor fundamental que permitirá el replanteamiento y restructuración que se requieren para hacer un proceso de branding dinámico, que se ajuste a las necesidades cambiantes del entorno competitivo (Cruz, 2012).

El objetivo general de este estudio fue explorar y describir el estado actual de los procesos de branding que efectúan las empresas tipo spin offy start up académicos, provenientes de las Universidades públicas en Colombia. Esto se llevó a cabo seleccionando e investigando el enfoque hacia el branding de las tres primeras empresas spin off académicas creadas en Colombia provenientes todas de la Universidad de Antioquia (en Medellín Colombia), y tres empresas más tipo start up académico: dos "provenientes" de la Universidad Nacional de Colombia sede Bogotá y una de la Universidad Distrital de Bogotá.

Otra investigación que tiene en cuenta el tema del Branding y de marca es el de Carolina Carreño Sánchez (2013) su investigación titulada: "Estudio diagnóstico sobre la gestión estratégica de la comunicación Marca-Ciudad de Santa Marta para el posicionamiento y visibilidad en los medios" Universidad Sergio Arboleda -Colombia. Propone la construcción de marca-ciudad mediante una estrategia de gestión de imagen a través de procesos comunicativos denominados branding, 
que promueva y contribuya al impacto en los públicos objetivos para generar reconocimiento, status, distinción, factor diferencial, reputación positiva, oportunidades de inversión y desarrollo económico (Carreño, 2013).

Entre las finalidades del estudio están el de analizar el mix de medios y las herramientas de comunicación utilizadas en el branding de la marcaciudad Santa Marta y el planteamiento de la propuesta estratégica para el fortalecimiento de la marca-ciudad.

\section{Discusión:}

El Branding ha tenido un papel muy importante y estratégico para el posicionamiento de las diferentes empresas y marca-ciudad presentadas en el capítulo anterior. $\mathrm{Y}$ ha sido primordial para la ampliación del mercado y en algunos casos en la internalización de estas nuevas marcas. Cúcuta Marroquinera. Uno de los ejemplos muy particulares de internacionalización de la marca a través del Branding es el proyecto "Estrategias Branding para el sector marroquinero de San José de Cúcuta, Norte de Santander" (Urbina y Palacios 2014). A través de la realización del diagnóstico al sector marroquinero de la ciudad de Cúcuta se conoció que es uno del más representativo y competitivo de la región con un alto dinamismo tanto a nivel nacional como internacional.

La ubicación geográfica del departamento Norte de Santander permite tener acceso al mercado interno y a los externos, a pesar de esto remplazar el mercado venezolano no ha sido fácil para los empresarios de la región sin embargo en el último año las exportaciones han tomado destinos diferentes a Venezuela, la producción de la industria de curtido de cueros y artículos de marroquinería mostraron un aumento significativo, esto muestra el gran esfuerzo de diversificación del mercado en las exportaciones de las empresas en la cadena productiva. (Urbina y Palacios 2014)

Con el diagnóstico realizado al sector marroquinero de la ciudad de San José de Cúcuta se conoció que cada uno de los empresarios tiene una visión muy importante en cuanto a posicionar sus productos en el mercado internacional y que de hecho a pesar de sus limitaciones en temas del marketing, los empresarios se han esforzado por aplicar estrategias que tienen a su alcance y que de alguna manera les han permitido en muchas ocasiones dar a conocer sus productos y venderlos en mercados internacionales.

Los esfuerzos que han tenido los empresarios del sector marroquinero de la ciudad de Cúcuta, les ha permitido el comercio con diferentes compradores internacionales, situación favorable para el dinamismo del sector y para darse a conocer el producto cucuteño, sin embargo ninguno de estos empresarios presenta un posicionamiento definido en ningún mercado internacional. Con la puesta en marcha de la marca representativa del sector marroquinero de la ciudad de San José de Cúcuta se tratará de destacar la importancia que tiene una marca apropiada, la cual transmita conceptos claros a través de los atributos y valores que posee el sector marroquinero de la ciudad y que se conocieron a través de la investigación realizada.

El diseño de la marca del sector consta de un logotipo y un eslogan que contienen los colores más representativos del sector, en este sentido se genera una imagen sólida y positiva a nivel nacional e internacional, y se afirma el sentimiento regional de optimismo que proyecta a los empresarios a posicionarse en cualquier mercado que se proponga.

Figura 2.

Marca: Imagotipo

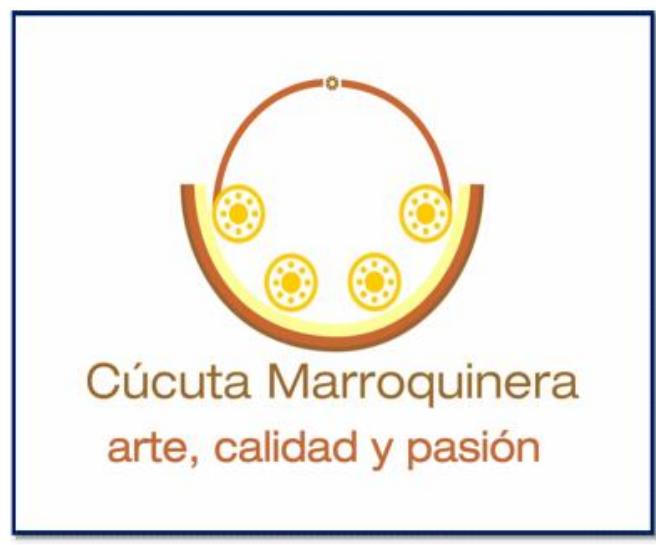

Fuente: Urbina y Palacios (2014)

Marca LDU Liga Deportiva Universitaria a nivel nacional: El resultado de aplicación del Branding evidenció la oportunidad de ampliación del mercado de los hinchas, porque el dar valor a la 


\section{LilianaMarcedaBastosCorio-JohamaMlenaMbgrovejoAndrade- LugycónezMna}

marca permitirá que los hinchas se identifiquen con la misma, a través de un vínculo racional y emocional por los beneficios que reciben, por lo tanto la idea a defender se confirma. Es así que el fútbol a través de la historia más que ser el deporte número uno en todos los países, ha sido una gran fuente de ingresos para los equipos. Esta inversión a la luz de un enfoque innovador de marketing deportivo permite poner en marcha una serie de estrategias que se han implementado en esta propuesta.

En esta primera parte del proyecto, sirvió para que en la ciudad de lbarra se validara la identificación de las necesidades de un mercado que no ha sido explotado por la marca, convirtiendo en una posibilidad de apropiación factible a aplicarla a nivel nacional. La estrategia establece un sistema de persuasión, que considera la imagen de los jugadores del equipo denotando éxito, liderazgo, victoria, fortaleza, decisión y completada con el slogan crea un vínculo racional y afectivo entre la marca y el hincha.

De acuerdo a la encuesta realizada los productos más demandados por los hinchas, fuera de los tradicionales son: $C D$, zapatos con firma, muñecos miniatura coleccionables, cartas coleccionables, entre otros. Por tanto estos productos pueden ser expendidos en un espacio identificado como "El Rincón Albo", el cual deberá estar localizado en Marathon Sports, proveedor directo del equipo. La propuesta considera diversos tipos de alianzas estratégicas con el uso de la tarjeta súper hincha que genera beneficios en: farmacias, restaurantes, línea de zapatos, servicios de salud, almacenes deportivos. (Ayala, J. 2009).

Ayala, (2009). plantea algunas recomendaciones para que sean tenidas en cuenta a la hora de implementar la marca, (Ayala, 2009):

- LDU es una entidad deportiva respetable, seria y prestigiosa por su trayectoria, con un gran equipo de futbol, circunstancia que debe ser aprovechada para aplicar el modelo planteado en el desarrollo de la presente tesis.

- Diversificar las alternativas de beneficios para los hinchas y facilidades para que este público llene sus expectativas.
- LDU debería implementar estas estrategias periódicamente a nivel regional, para a futuro intervenir en el mercado internacional. Los reconocimientos internacionales son importantes para implementar valor a la marca LDU.

- Es importante que LDU nunca pierda de vista las necesidades del cliente y brindarle muchas opciones en cuanto a promociones, facilidades de compras, alianzas estratégicas, entre otras.

- Mediante la implementación de este modelo, LDU podrá mantener la fidelidad de los hinchas y ellos sentirse orgullosos de la organización que los representa, creando un incentivo hacia el target que queremos dirigirnos, yendo siempre un paso delante de la competencia.

- LDU debería implementar un plan de Relaciones Públicas para reforzar la puesta en marcha del nuevo modelo. (Ayala, 2009)

Brandig y marcas para empresas spin off y start up académico de las Universidades Públicas en Colombia. Se estableció que las investigaciones de marca y branding se han realizado especialmente para grandes compañías, y que para las PYMES es un tema que aún se encuentra en sus estados iniciales. Dentro de las PYMES, una categoría de creciente importancia en Colombia, son las compañías tipo spin off y start up académico. Aunque algunas investigaciones de marca $y$ branding se han realizado para PYMES, en sectores como manufactura, comercio electrónico, organizaciones sin ánimo de lucro, software y servicios, ninguna ha sido llevada a cabo en spin offsy start up académicos. (Cruz, 2012).

Dentro de esta reflexión de los estudios de marca y branding para PYMES, se determinó que las variables de branding que más se adaptan a las spin off y start up académicas son las que comprenden: Relevancia de la marca: importancia percibida por los emprendedores sobre las actividades de branding, incluida la plataforma de marketing necesaria, conocimiento de conceptos y aspectos de gestión corporativa; Creación de marca: comprende los componentes de la identidad de marca, enfocados al aspecto visual y gráfico de la misma; y Construcción de marca: actividades que 
permiten el desarrollo de la marca y la construcción de su imagen. (Cruz 2012)

Igualmente identificó que las prácticas de branding que han tenido en cuenta las empresas spin off y start up académico de las Universidades Públicas en Colombia comprenden (Cruz 2012):

No tienen un departamento o un responsable formal de mercadeo, sino que las actividades concernientes están distribuidas entre todos los miembros de la empresa.

Dan prioridad a la creación del nombre de marca (naming), del logo símbolo y el registro legal de los mismos.

Emplean la publicidad (menciones gratuitas en medios) como instrumento favorito de anunciarse, en razón a la novedad y el prestigio académico que tienen.

Captan clientes empleando el voz a voz y la red de contactos como medios principales, los cuales constituyen también actividades de marca.

El emprendedor en sí mismo es una "representación viva" de la marca.

El personal que seleccionan para trabajar en sus empresas proviene o tiene relación con el mundo académico.

El respaldo de marca de la universidad de la que provienen es altamente relevante (al menos en sus estados iniciales), en especial para las spin off, puesto que pueden emplear dicho respaldo "abiertamente".

El Branding es una buena estrategia para el posicionamiento comercial y no solo ha sido implementado a nivel de empresas, sino también a nivel de Industrias como lo plantean para el sector de Marroquinería de la ciudad Cúcuta. De esta manera, con la aplicabilidad de este modelo se está explorando nuevos mercados los que se hacen necesarios para internacionalizar los productos de la región, el cual ha sido bastante afectada por la disminución comercial con el vecino país de Venezuela.

Es así que se requieren de este tipo de alternativas comerciales y estratégicas, no solo para consolidar un mercado sino también para posicionar una marca o un producto en el exterior. A través de este trabajo se hizo posible la reflexión de ocho trabajos de investigación, los cuales proponen para captar mercados y quedarse dentro de las preferencias de los consumidores, la teoría del Brandig, como se muestra a continuación:

Tabla 2.

Relación de proyectos de investigación aplicando el Branding como estrategia comercial.

\begin{tabular}{|c|c|c|}
\hline Nombre del Proyecto & $\begin{array}{l}\text { Tema ó sector } \\
\text { económico }\end{array}$ & Autores \\
\hline $\begin{array}{l}\text { Estrategias branding para el } \\
\text { sector marroquinero de San } \\
\text { José de Cúcuta, Norte de } \\
\text { Santander. } 2014\end{array}$ & Marroquinería & $\begin{array}{l}\text { Ciro Urbina } \\
\text { y Julián } \\
\text { Palacios }\end{array}$ \\
\hline $\begin{array}{l}\text { Esquema de posicionamiento } \\
\text { y persuasión para la marca } \\
\text { LDU a nivel nacional, } \\
\text { tomando como plan piloto la } \\
\text { ciudad de Ibarra. Año } 2009\end{array}$ & Deportivo & $\begin{array}{l}\text { José Luis } \\
\text { Ayala Rivero }\end{array}$ \\
\hline $\begin{array}{l}\text { "Branding en Chile, } \\
\text { Universidad de las Américas. } \\
2007\end{array}$ & $\begin{array}{l}\text { Alimentos y } \\
\text { Marca-Ciudad }\end{array}$ & $\begin{array}{l}\text { Peter Brill y } \\
\text { Mauricio } \\
\text { Pradenas }\end{array}$ \\
\hline $\begin{array}{l}\text { Las Marcas cambian de piel, } \\
\text { pero no pierden su esencia"- } \\
\text { Pontificia Universidad } \\
\text { Javeriana, Bogotá - } \\
\text { Colombia. Año } 2009\end{array}$ & Automotriz & $\begin{array}{l}\text { Natalia } \\
\text { Lema y } \\
\text { Adriana } \\
\text { Peláez }\end{array}$ \\
\hline $\begin{array}{l}\text { Construcción de la identidad } \\
\text { de marca para la empresa } \\
\text { calzado ROGGER'S LTDA. - } \\
\text { Universidad Autónoma del } \\
\text { Occidente - Valle del Cauca - } \\
\text { Colombia. } 2011\end{array}$ & Calzado & $\begin{array}{l}\text { Diana Hoyos } \\
\text { Forero }\end{array}$ \\
\hline $\begin{array}{l}\text { "Estado actual del branding } \\
\text { en las compañías spin off y } \\
\text { start up académico: caso } \\
\text { universidades públicas en } \\
\text { Colombia - Universidad } \\
\text { Nacional de Colombia, } \\
\text { Bogotá. } 2012\end{array}$ & Educación & $\begin{array}{l}\text { Jonnathan } \\
\text { Jair Cruz }\end{array}$ \\
\hline $\begin{array}{l}\text { "Estudio diagnóstico sobre la } \\
\text { gestión estratégica de la } \\
\text { comunicación Marca-Ciudad } \\
\text { de Santa Marta para el } \\
\text { posicionamiento y visibilidad } \\
\text { en los medios". } 2013\end{array}$ & Marca- Ciudad & $\begin{array}{l}\text { Carolina } \\
\text { Carreño } \\
\text { Sánchez }\end{array}$ \\
\hline $\begin{array}{l}\text { Diseño de un plan de } \\
\text { marketing estratégico para } \\
\text { empresas de servicios } \\
\text { temporales EST basado en el } \\
\text { diagnóstico de medianas y } \\
\text { grandes empresas del sector } \\
\text { comercial" - Universidad } \\
\text { Nacional de Colombia sede } \\
\text { Manizales. } 2008\end{array}$ & Servicios & $\begin{array}{l}\text { Francy } \\
\text { Milena } \\
\text { Aguirre }\end{array}$ \\
\hline
\end{tabular}




\section{LilianaMarcelaBastosGorio-JdhennaMlenaMborovejoAndrade- LugyCónezMna}

\section{Referencias:}

Alvarado, U. (2013). Tabla: Infografico Antecedentes Históricos del Marketing y la Gestión de Marcas. Recuperado de: http://www.iuriel.com/mercadotecnia/tabl a-infografico-antecedentes-historicos-delmarketing-y-la-gestion-demarcas/(Recuperado el 20 julio 2014)

Andema. (2011).¿Qué son las marcas? Recuperado de: $\quad$ http://andema.camaras.org/?q= content/que-son-las-marcas

Arango, A. (2009). Entrevista con David Clifton, Director General de Interbrand México. Recuperado de: http://segmento.itam.mx/Administrador/U ploader/material/Entrevista\%20con\%20Da vid\%20Clifton-Interbrand.pdf

Arango, A. (2009). En que consiste la teoría del branding. Recuperado de: http://rincones pecialista.blogspot.com/2010/08/en-queconsiste-la-teoria-del-branding.html

Asociación Nacional para la Defensa de la Marca. (2013). ¿Qué son las marcas? Recuperado de:

http://andema.camaras.org/?q=content/qu e-son-las-marcas.

Ayala Rivero, J. (2009). Propuesta de un esquema de posicionamiento y persuasión para la marca LDU a nivel nacional, tomando como plan piloto la ciudad de Ibarra. Tesis de grado, Quito, Universidad Tecnológica Equinoccial.

Bernardos, L. (2012). Hablemos con propiedad, parte 2: los términos estratégicos de una marca. Recuperado de: http://www.brandemia.org/hablemos-conpropiedad-parte-2-los-terminosestrategicos-de-unamarca\#sthash.8rBvF0kY.dpuf

Brainbox. (2008). El broading y sus principios. Recuperado de: http://brainboox.com/elbranding-y-sus-principios/

Brill Ramírez, P. y Pradenas, M. (2007). Branding en Chile. Tesis de grado, Santiago de Chile, Universidad de las Américas. http://es.scribd.com/doc/148876971/Tesis-deBranding-en-Chile

Carreño Sanchez, C. (2013). Estudio diagnóstico sobre la gestión estratégica de la comunicación Marca-Ciudad de Santa Marta para el posicionamiento y visibilidad en los medios. Tesis de grado, Bogotá, Universidad Sergio Arboleda.

Cruz, J. (2012). Estado actual del branding en las compañías spin off y start up académico: caso universidades públicas en Colombia. Tesis de grado, Bogotá: Universidad Nacional de Colombia.

Gobernación de Norte de Santander. (2013). Información general. Recuperado de: http://

www.nortedesantander.gov.co/infgeneral. php

Hoyos, D. (2011). Propuesta de construcción de la identidad de marca para la empresa calzado ROGGER'S Ltda. Tesis de grado, Cali, Universidad Autónoma del Occidente.

Kloter, P. y Armstrong, G. (2009). Fundamentos de Marketing. México: Prentice Hall.

La Base. (2009). Ciclo de vida de la marca. Recuperado de: http://www.labase.cl/ciclode-vida-de-la-marca/

La Republica. (2013). Los productos chinos son una 'papa caliente'. Recuperado de: http://www.larepublica.co/los-productoschinos-son-una-\%E2\%80\%98papacaliente\%E2\% 80\%99_41068

Lema, N. y Peláez, A. (2009). Marcas en cuerpo y alma. Tesis de grado, Bogotá, Pontificia Universidad Javeriana.

Luna, D. (2011). Revisión de la literatura. Recuperado de: http://catarina.udlap.mx/u_dl_a/ tales/documentos/lhr/luna_d_a/capitulo2. pdf

Nito, M. (2014). El valor de una marca. Recuperado de: http://www.soyentrepreneur.com/marketi ng-670.html

Sánchez, B. (2013). La marca I: Concepto y objeto. Recuperado de: http://sanchezbermejo.com/la-marca-iconcepto-y-objeto/

Thompson, I. (2006). Concepto de empresa. Recuperado de: http://www.promonegocios.net/ empresa/concepto-empresa.html.

Urbina, C y Palacios, J (2014). Estrategias Branding Para El Sector Marroquinero De San José De Cúcuta, Norte De Santander. Trabajo de grado UFPS, Cúcuta. 